\title{
REFLEXIONES EN TORNO AL MODUS OPERANDI DE LA FRANQUICIA EN EL DEPORTE
}

\section{Francisco Javier Pérez-Serrabona González}

Profesor de Derecho Mercantil de la Facultad de Derecho de la Universidad de Granada email: jpsgonzalez@ugr.es

RESUMEN :Nuestra reflexión va encaminada a mostrar otros modelos de negocio deportivos, revestidos jurídicamente con los contratos de "franquicia" y sus distintas modalidades, y con un éxito basado en el binomio deporte-rentabilidad que quizás deberíamos plantearnos en un futuro no muy lejano, no ya con dicho modelo en su vertiente más pura, pero si al menos con alguna fórmula jurídica híbrida entre la descrita y la actual, que permitiese obtener beneficios generando el mejor espectáculo deportivo, y sobre todo, ajustando el deporte a las nuevas tendencias y necesidades del mercado.

PALABRAS CLAVE: Franquicia, clubes, principio de equidad, asesoramiento, holding, competencia.

\begin{abstract}
Our paper is aimed at showing other sports business models, covered with contracts legally "franchise" and its various forms, and with a success based on the binomial sport-profit that perhaps we should consider in the not too distant future, no longer with the model in its purest aspect, but at least with some hybrid between the legal formula described and the current, which allow profit generating the best sporting event, especially by adjusting the sport to new trends and market needs .
\end{abstract}

KEYWORDS: Franchise, clubs, principle of equity, advice, holding, competition 


\section{I.- LA FRANQUICIA EN EL DEPORTE: OTRA FILOSOFÍA COMERCIAL.}

Indudablemente el acceso a nuevos mercados internacionales es un paso en la evolución de la mayoría de redes de franquicia, pero esta evolución requiere de una preparación en diferentes ámbitos y una adaptación de distintos elementos. A las necesidades habituales del sistema de franquicia hay que unir las específicas y particulares de cada mercado internacional, tanto en el ámbito societario, como en el relativo al sector de actividad en concreto o a la regulación de franquicias. La franquicia en el sector del deporte creemos que tiene un campo de acción y una perspectiva de negocio en constante evolución, y que sin duda se asentará y consolidará en breve espacio de tiempo al igual que lo ha hecho en otros países.

Así, a modo de ejemplo destaca Estados Unidos, donde desde hace ya muchos años el deporte profesional se organiza mediante el sistema de franquicias ${ }^{1}$. La implantación de este modelo en el deporte americano es sencilla ${ }^{2}$; el organizador de las distintas ligas deportivas -profesionales-, admite distintos participantes bajo la condición de que cumplan aspectos económicos (pago de unos cánones) para que sean sometidos a una regulación de normas específicas de esa franquicia, sin relegar equipos o con sistemas de promoción de campeonatos, como el caso que sucede en Europa y Sudamérica. Las ligas americanas no pretenden satisfacer objetivo alguno de ninguna organización superior a ellas, estas ligas surgieron fruto de la iniciativa empresarial que buscaba obtener la máxima rentabilidad económica de sus espectáculos creando un modelo de negocio. Por lo tanto el objetivo claramente en América consiste -y así fue concebido-, en ser un modelo económicamente rentable, y donde se buscaba, no ya sólo

\footnotetext{
${ }^{1}$ Este término se emplea aquí como sinónimo de Club, entidad deportiva o empresa deportiva, si bien en su acepción técnica en el marco de las reglas de las Ligas Profesionales adquiere un sentido más cercano al atribuido en el tráfico mercantil, como equivalente a derecho exclusivo reconocido por una Liga a una empresa deportiva para operar en un marco territorial, sin que concurra la competencia de ninguna otra salvo autorización de la Liga. (Cfr. R. TEROL GómeZ, R., Las Ligas Profesionales, Edit. Aranzadi, 1998, pág. 49).

${ }^{2}$ La NBA, es una Liga creada en 1946 por un grupo de empresarios decididos a unificar las dispersas ligas de baloncesto para crear un único modelo donde se concedió acceso a 11 franquicias, que con los años han elevado su cifra hasta los 30 actuales, y esta basado en un modelo eminentemente "empresarial" (Cfr. FORT, R., "Revenue disparity and competitive balance in Major League Baseball", Informe año 2000 para el Comité antitrust del Senado de los EE.UU).
} 
la especialización de los deportistas, sino la explotación de una industria denominada del entretenimiento. ${ }^{3}$

El modelo de las ligas mayores americanas pivota sobre el concepto de la franquicia. La franquicia se fundamenta en estas ligas como la concesión que otorga la Liga a una determinada sociedad, habilitándola para explotar un equipo que participe en la competición, y vinculándola a un determinado ámbito territorial - home territory -, sobre el que se otorgan determinados derechos exclusivos, a fin de asegurar la viabilidad económica del proyecto empresarial del -potencial- franquiciado.

Debemos destacar que dentro de este sistema de franquicia deportiva americana, nos encontramos con un modelo de negocio y de organización deportiva absolutamente privado, ya que la actuación de los poderes públicos es mínima y no existe a nivel Federal, ningún organismo público que tutele el fenómeno deportivo, al igual que tampoco existe cuerpo legislativo cohesionado acerca del deporte.

La base en la que se sustenta este modelo es la convicción que se tiene en América de que el deporte profesional es un asunto exclusivamente privado, por lo que la aplicación integra del ordenamiento jurídico privado a esta actividad como a cualquier otra actividad empresarial resulta pacífica. Clara exposición de este fenómeno es que las denominadas "Major Leagues", son el resultado de sucesivos procesos de concentración empresarial, en el que algunas ligas han ido incorporando mediante absorción a otras ligas ${ }^{4}$.

\footnotetext{
${ }^{3}$ En este sentido destacamos la Sentencia Chicago Professional Sports Limited Partnership \& WGN $v$. NBA (1992, 961 F.2d 667), cuando refiriéndose a la Liga de Baloncesto americana (MBA) expresa: "el baloncesto profesional en televisión no es fundamentalmente diferente de la serie televisiva Star Trek: The Next Generation" produce igualmente "entretenimiento", y esta finalidad informa además de todos los aspectos de la competición, desde el sistema de elección de los jugadores - los conocidos drafts, que buscan el equilibrio entre los equipos -, hasta la adaptación de las reglas deportivas en búsqueda del espectáculo - como los tiros triples, implantados por primera vez en la American Basketball League (hoy incorporada en la NBA) y luego adoptados por la FIBA-.

${ }^{4}$ Ejemplos ilustrativos de las mismas son: la National Football League (NFL), la Major League Baseball (MLB), la National Basketball Association (NBA) y la National Hockey League (NHL). Se tratan de modelos de negocio elaborados conjuntamente entre franquicias -clubes-, competidores en el terreno deportivo, pero obligados a la colaboración inter-empresarial. (Cfr. FREEDMAN, W.,Professional sports and antitrust, Quorum books, New York, Wesport Connecticut, London, 1987, pág.5).
} 


\section{CLUBS O FRANQUICIAS; DEPORTE O NEGOCIO}

Estamos ante un negocio -deportivo-, que está sometido estrictamente a las normas del comercio, y en el que se generan los mismos conflictos que pueden reproducirse en cualquier actividad del ámbito empresarial, por lo tanto son normas generales en los mismos: las relaciones contractuales de equipos, los jugadores (personal trabajador), los gestores (del patrimonio de las franquicias), los innumerables acuerdos contractuales de patrocinio, publicidad, etc., éstos acuerdos, no están exentos de polémica como cualquier actividad o entorno laboral, por lo que se resolverán de la misma manera y conforme a las mismas normas que el resto de las actividades del mundo empresarial. ${ }^{5}$

Tal ha sido el éxito americano, que el modelo se extendió a las fronteras del los Estados Unidos, en concreto en las grandes ligas en Canadá ${ }^{6}$, e incluso en la actualidad, estas ligas americanas están estudiando su expansión empresarial y la muestra de su negocio por toda Europa (al margen de las instituciones deportivas y federativas nacionales que están implantadas en los organismos internos de cada país y europeos). ${ }^{7}$

\footnotetext{
${ }^{5} C f r$. los trabajos realizados en este sentido por; PÉREZ DE LOS COBOS ORIHUEL, F., "Mecanismos legales de descuelgue salarial", La aplicación de la reforma del Estatuto de los Trabajadores en la negociación colectiva, IX Jornadas de Estudio sobre la Negociación Colectiva, Ministerio de Trabajo y Asuntos Sociales. Madrid, 1996; RUBIO SÁNCHEZ, F., El contrato de trabajo de los deportistas profesionales, Dykinson, 2002; IRURZUN UGALDE, K., "Convenios colectivos y deporte profesional", $R E D D$, Edit. Civitas, $\mathrm{N}^{\circ}$ 6, 1995; GARCÍA SILVERO, E. A., "Hacia una nueva regulación de los traspasos de los deportistas profesionales en la UE", Boletín Mensual Aranzadi Laboral, $\mathrm{n}^{\circ}$ 13, 2000; CARDENAL CARRO, M., Deporte y Derecho: las relaciones laborales en el deporte profesional, Universidad de Murcia, 1996; CAZORLA PRIETO, L. M., Derecho del deporte, Edit. Tecnos, 1992.

${ }^{6}$ Con especial referencia a la National Hockey League (NHL), que dentro de su deporte, es la liga más importante del mundo, según la Federación Internacional de Hockey sobre Hielo. Con la apertura del campeonato de Europa Occidental y Europa del Este, su importancia a nivel internacional ha aumentado y se ha convertido en destino preferente para jugadores de otros torneos. La National Hockey League es una de las cuatro ligas más importantes en la cultura deportiva norteamericana, junto a la del futbol americano, la de Beisbol y la de Baloncesto. El campeonato ha aumentado sus participantes con el paso del tiempo, y actualmente comprende 30 franquicias. De ellas, 7 están situadas en Canadá y 23 en Estados Unidos.
}

${ }^{7}$ En el caso del futbol en España debería de plantearse, si no ya este modelo de negocio estructurado, franquiciado, donde en definitiva prime más la rentabilidad que lo meramente deportivo, si al menos un sistema híbrido que no fuera tan deficitario, ya que hasta ahora, los clubes de futbol y baloncesto, se permitían fichajes millonarios y presupuestos ficticios basados en los derechos de televisión, que ya no parecen tener el éxito y la repercusión esperados cuando las principales operadoras han empezado a recortar las condiciones económicas contractuales, debido a las pérdidas de audiencia y pérdidas económicas que provocan estas actividades deportivas en los principales medios de difusión. El retransmitir partidos no sale tan rentable -al menos fuera de los equipos "grandes"-, y la consecuencia inmediata es que la estructura del fútbol y baloncesto en España -como ocurre en la mayoría de los 
El modelo de franquicia que tienen implantando es un sistema cerrado, cuya característica esencial es la estabilidad de todos los participantes, esto es, las competiciones buscan asegurar estabilidad y rentabilidad de los participantes, y quizás olviden, los méritos deportivos de cada uno, de esta forma no contemplan los ascensos y descensos. ${ }^{8}$

\title{
III. MODELO AMERICANO DE FRANQUICIA DEPORTIVA
}

Como sabemos el sistema de franquicia es, ante todo, una filosofía, una filosofía comercial, porque se unen, al mismo tiempo, la voluntad conjunta de prosperar con la aportación de las habilidades y valores de cada parte; voluntad, habilidades y valores que, conjuntándose armoniosamente, conseguirán el éxito de la actividad empresarial. Es un negocio mutuo, en el que el franquiciador cuando le falta capacidad económica o

\begin{abstract}
escenarios europeos-, o cambia, o pasará por situaciones no deseables, como ya ha sucedido durante estos años con la quiebra de varios equipos históricos y emblemáticos del futbol y baloncesto español. Curioso es resaltar de la misma manera -y a colación con lo señalado-, el sistema tan diferente para televisar la distintas Ligas en EEUU, así en baloncesto por ejemplo, la NBA, los derechos televisivos que le reportan a las franquicias, actualmente son de 930 millones de dólares, pero el reparto de dichos derechos curiosamente-, se hace absolutamente en la equidad para cada uno de los treinta equipos de la liga que la compone., luego cada equipo -franquicia-, ingresará 31 millones al año, sin preferencias ni distinciones. Caso absolutamente contrario sucede en la Liga española, por ejemplo, de futbol (también llamada liga BBVA), donde en la temporada presente 2014-15 el Barcelona y el Real Madrid ingresarán 150 millones de euros cada uno, mientras que habrá equipos que no lleguen a los 3 millones de euros por derechos televisivos.
\end{abstract}

\footnotetext{
${ }^{8}$ Para evitar las desigualdades existentes en una temporada, priman en lo deportivo a los peores equipos peores franquicias-, para que con vistas a la siguiente temporada puedan beneficiarse en los procesos de selección de nuevos jugadores, éstos, son los denominados "drafts", formados en la NCAA (la liga universitaria), competición en la cual un jugador de universidad solo la puede jugar cuatro años, que es el tiempo en que en Estados Unidos se tarda en obtener una licenciatura, una vez ingresado y según que deporte practique cada jugador es alojado dentro del "draft" que le corresponde a la liga profesional de su deporte (si es futbol americano se van al draft de la NFL, si es beisbol, al draft de la MLB, si es al soccer (nuestro futbol), al de la MLS; si es hockey sobre hielo al de la NHL y si es básquetbol al de la NBA). Los jugadores que surgen del torneo universitario al término de esos cuatro años son escogidos según su nivel, y cuanto mas alta es la posición, mayores posibilidades tendrán de ser contratados por la liga profesional al año siguiente. No obstante, el draft de la NBA con el tiempo -y esto es debido a que el baloncesto es un deporte practicado por todo el mundo- se convirtió en un "mercado" que obedece a su lógica, permitiendo año tras año que cada vez mas jugadores de fuera, que juegan en clubes FIBA, se inscriban masivamente al sorteo junto a los colegiales. Lo curioso de este modelo de negocio, es que prima a la hora de elegir a este tipo de jugadores el orden inverso a como han finalizado los equipos la competición ese año, y desde el punto de vista contractual, los seleccionados del draft pueden ser tranquilamente intercambiados como parte de pago en las transferencias internas de los jugadores. Los equipos además retienen sus derechos sobre sus jugadores escogidos si alguna vez desean emigrar. De dicha forma se consigue igualmente la máxima de que toda franquicia pueda competir en igualdad de condiciones, y que toda franquicia deportiva como sistema comercial y empresarial que constituye, prosperen con mayor facilidad a lo largo del tiempo.
} 
de control para organizarlo todo por sí solo, aporta de modo permanente su técnica y método al franquiciado; y por otro lado, el franquiciado, recibe esa técnica y ese método para que su negocio funcione y sea rentable. Pues dicha paradoja y esencia del modelo de franquicia, es el que aplica la National Basketball Association o NBA (Asociación Nacional de Baloncesto), principal liga estadounidense de baloncesto profesional, fundada en Junio de 1946 y principal ejemplo de filosofía comercial deportiva objeto de negocio franquiciable. Se trata de una entidad totalmente independiente $y$ completamente autogestionable, que se desempeña de manera privada y al margen de la federación americana (US Basketball), y con el modelo de franquicia como principal baluarte de sus acuerdos contractuales. Así, los equipos que conforman dicha entidad Liga-, son franquicias, que a su vez son corporaciones en sí mismas: es decir, que estamos hablando de autenticas sociedades anónimas que constituyen un gigantesco holding, en donde la Liga-NBA-, está configurada como una sociedad limitada, y en la que sus socios -o propietarios-, son los equipos que operan en régimen de franquicia. Como tal modelo de franquicia, cada equipo que quiera entrar en la liga deberá pagar antes un canon -en este caso de alrededor de 300 millones de dólares a la propia sociedad gestora-, y una vez realizado el mismo -la inclusión no es ni mucho menos inmediata, ya que cada equipo debe esperar unos cinco años antes de saber si finalmente ingresa-, esperará un tiempo en donde su equipo será sometido a una serie de auditorías contables para comprobar la transparencia de estas nuevas empresas -que son futuras franquiciadas para integrarse a la red franquiciadable-. El siguiente paso que deberá realizar el potencial franquiciado, será ofrecer a la sociedad gestora un plan de negocio, para que se evalúe su viabilidad económica y deportiva.

Una vez aceptados e integrados en dicha red, cada franquicia se encargará de todos los contratos de difusión y comunicación a nivel local, pero será la Liga en sí, la que se reserve el derecho de todos los contratos firmados a nivel nacional. La Liga centralizará el merchandising y los acuerdos de partnership ${ }^{9}$, al mismo tiempo que distribuirá los dividendos a los clubes de manera proporcional ${ }^{10}$, igualmente será la que

\footnotetext{
${ }^{9}$ Partnerschip es un acuerdo entre dos o más personas para compartir la operación y ganancias de una gestión comercial que no es incorporada.

${ }^{10}$ Recientemente (7/10/14, paginan web oficial; www.nba.com), es significativo el anuncio de la franquicia NBA anunciando la extensión del contrato de explotación de sus derechos televisivos hasta la temporada
} 


\section{Doctrina y Jurisprudencia}

determine el modo de explotación de los recursos, disponiendo, por ejemplo, la explotación colectiva o individualizada de los derechos de retransmisión audiovisual de los partidos, y finalmente será la que fije el reparto de los ingresos obtenidos por taquilla, y la que derive de su condición de patronal la intervención sobre el mercado laboral de cada uno de sus franquiciados - o clubes integrantes de su red-, fijando la distribución de sus rentas de la industria.

Resumiendo, nos encontramos con un peculiar modelo de franquicia deportiva el que en la actualidad está implantado en América, que podíamos definirlo como un tipo de marca que se dedica al deporte y que, además de ganar el título, busca obtener beneficios como empresa, y en la cual no existe el concepto de accionistas, y si, el de propietarios. Su entidad deportiva dependiente -La liga-, administra el número de participantes en la misma -el número de franquicias aceptadas-, contando de esta forma con un número cerrado de participantes que optan al título, y en el que todos, absolutamente todos, dependen de la propia entidad deportiva -esto es, de la Liga-; y es tal la relación de dependencia entre ambas partes que integran el negocio, que todas las decisiones de relevancia que quieran implantar cada una de las franquicias individualmente, deberán ser respaldadas por la propia Liga y por el resto de las franquicias deportivas integrantes de la competición.

De esta forma, como podemos observar el principio de equidad rige en toda la competición y en cada una de las franquicias integrantes de la misma. Así a modo de

2024/25, acuerdo en torno a 24.000 millones de dólares (aproximadamente 19.000 millones de euros por nueve temporadas), observemos la importancia con este monto que tiene el modelo de franquicia americano implantado y que a buen seguro debe de ser tomado en cuenta por otros modelos mundiales europeos, e incluso nos atrevemos a decir español-. Indudablemente este incremento repercutirá en cada una de sus 30 franquicias actuales ya que pasará a ingresar de los 930 millones de dólares actuales a cerca de 3.000 por temporada, el cual se repartirá completamente en equidad entre las tantas franquicias pertenecientes a la competición. Igual repercusión tendrá en el actual Convenio Colectivo el cual establece que los jugadores deben recibir el 51\%de los ingresos que genera la competición, por lo que el impacto sobre los mismos, será evidente, ya que se pasará del límite salarial de 63 millones actuales por temporada a cerca de 80 millones de dólares. Los jugadores, principal baluarte de dichas franquicias, aspirarán a un contrato máximo, que se calcula como un porcentaje máximo del limite salarial (en el caso de los jugadores con más de 10 años de experiencia por ejemplo este tope estaría en torno al 35\%), luego el no renovar por algunos de ellos en la actualidad, les podría suponer un aumento potencial muy elevado del beneficio con la firma del nuevo Convenio. Afirmamos pues, que este modelo de negocio -franquiciable-, ha adquirido una rentabilidad más que aceptable para el deporte americano, para sus jugadores, y sobre todo, a los consumidores y usuarios del mismo -al publico en general-, con el espectáculo y el "entretenimiento" que éste le reporta, y que no hace más que cerciorar que para las nuevas tendencias y necesidades del mercado deportivo, este nuevo modelo de negocio adaptado a este entorno, se convierte en uno de los contratos que mayor éxito empresarial y mayor repercusión mundial tendrá en tiempos venideros para el deporte internacional. 
ejemplo en cuanto a su retribución, todas las franquicias tendrán acciones igualitarias en MLB Advanced Media ${ }^{11}$, y la organización de dicho modelo se concebirá pues, como una "Limited Corporation" cuyos socios (los equipos, actualmente treinta), son franquicias, que a su vez son corporaciones en sí mismas y serán como decimos, auténticas sociedades anónimas las que constituyen este gran holding.

Las ventajas de este modelo están quedando patentes, pero creemos que la base de su éxito se sustenta en dos elementos fundamentales; en primer lugar, la ya citada "equidad" entre todos sus participantes, la cual juega un rol primordial base para la estabilidad y el futuro de dicha competición ${ }^{12}$; y en segundo lugar el binomio inseparable de este negocio de elaborar un producto deportivo, cuya premisa principal y necesaria para la estabilidad del negocio, la conforma el ser un modelo atractivo $-y$ de entretenimiento- para los consumidores y usuarios -verdaderos beneficiarios-, y en consecuencia, un modelo deportivo rentable para la franquicia y para sus franquiciados ${ }^{13}$.

\footnotetext{
${ }^{11}$ Entre otras cosas, esta empresa de videojuegos y aplicaciones para aparatos electrónicos y digitales, ha generado ingresos millonarios por sus vídeos juegos y aplicaciones, como el "MLB At Bat", aplicación deportiva de mayor descarga mundial para iPhone y los iPad desde 2010, y ya lleva cuatro años en primera fila.
}

${ }^{12}$ Aunque dicho tema de la equidad creemos que debería reformarse y regularse más profundamente ya que, observando los movimientos contractuales de alguna franquicia, podría provocar ciertas "suspicacias" en algunos equipos, sacrificando temporadas en la elección de jugadores -con los consiguientes resultados negativos-, para en el futuro, y al disponer de mayor porcentaje de distribución de jugadores franquicia y jugadores del "draft", convertirse en el mejor bloque deportivo, y con unas garantías de estabilidad en lo más alto de la competición a lo largo de varios años muy elevadas. El sistema actual del denominado "draft" es un sistema que premia con más opciones de conseguir una elección alta de estos jugadores "revelación", más destacados y con mejores promedios, a los equipos con peor balance de victorias, y ello podría provocar que una franquicia construyese -aún asumiendo el riesgo que ello le provocaría en una temporada-, ser la peor franquicia deportiva una temporada, para asegurarse elegir a la mayores figuras (ya promesas) del "draft" el siguiente año y cumplir su objetivo de permanecer en los más alto de la competición durante años, ya que supuestamente podría constituir el mejor equipo totalmente renovado, y con unos jugadores de tal envergadura que muy difícilmente en un mercado abierto podría conseguir. Luego pensamos que deberían de ahondar más en la regulación del sistema, y que cayeran en desuso iniciativas de este tipo, donde el principio de la buena fe contractual, quedaría totalmente en entredicho.

${ }^{13}$ Vid. en este sentido los trabajos realizados por; FERNÁNDEZ, M. y MARTíN, J., "La confianza y el compromiso como factores clave de éxito en las relaciones comerciales. Una aplicación empírica en el sistema de franquicia", Revista Europea de Dirección y Economía de la Empresa, Vol. 15. №.1, 2006, págs.77-100; FIZEL, J.L.,"Free agency and competitive balance", en Stee-rike four, (Coord. MARBURGER, R.), Edit. Praeger, 1997, págs.61-72; BUTLER, M.R., "Competitive balance in MLB", The American Economist, Vol. 39, № 2, 1995. 


\section{DIFERENCIAS ENTRE EL MODELO DE NEGOCIO EUROPEO Y} AMERICANO

La principal diferencia de los distintos modelos de negocio deportivos europeos $\mathrm{y}$ americanos, es que aunque existe un modelo único de contrato y unas "bases y condiciones" que han de ser aceptadas de manera generalizada si se quiere entrar en la organización en ambos modelos de negocio, en las competiciones deportivas europeas, dicho contrato se firma con la propia Liga, y es ésta, quien luego cede al equipo en cuestión la responsabilidad de pagarle el sueldo estipulado en contrato, organizando cada uno de los club sus contratos y los pagos de salarios. ${ }^{14}$ Mientras que en el modelo americano, los contratos y transferencias de los jugadores no los manejan las franquicias -o clubs-, sino que lo hace la propia Liga, y es ésta, la que le atribuirá a todas las franquicias por igualdad un salario equivalente a repartir entre sus contratos con los jugadores, el denominado "tope salarial".

En el modelo americano igualmente, como en todo contrato de franquicia, cada equipo que desee ingresar -en la cadena franquiciada-, deberá depositar a la liga un canon. Este canon será de abono obligatorio para pertenecer a la franquicia (y por ende, para competir en dicha Liga). En la misma cada jugador tiene un valor que representa exactamente lo que este cobra de sueldo en el año en curso ${ }^{15}$. Cuando se produce una transferencia de jugadores entre dos equipos de la NBA, lo único que tienen que hacer es "intercambiar" jugadores que representen un monto similar de dinero. El dinero en

\footnotetext{
${ }^{14} C f r$. BERMEJO VERA, J., "El marco jurídico del deporte en España", RAP, no 110, 1986; MONTES FLORES, V., "Los derechos federativos y su contenido patrimonial", Ponencia I Encuentro de Máster en Derecho Deportivo de la Universidad de Lleida, Marbella, 30 de mayo de 2002. (En internet, www.sportsciencies.com/); ALONSO MARTíNEZ, R., "La política europea de protección de los jóvenes deportistas", http://www.efdeportes.com/ Revista Digital, No 39 - Agosto de 2001; CARDENAL CARRO, M., Deporte y Derecho: las relaciones laborales en el deporte profesional, Universidad de Murcia, 1996.

15 Actualmente existe un Convenio (2014) que señala las normas de funcionamiento para los jugadores potencialmente integrantes de la competición, el cual establece un limite de edad para los jugadores -19 años-, para entrar a formar parte del "draft", y una exigencia para los mismos consistente en que dichos jugadores deben pasar además un año desde la graduación en el Instituto, lo que en la práctica les obliga a pasar por la Universidad. El gran inconveniente que se encontraban era que hasta ahora las normas prohibían pagar a esos deportistas -que en la mayoría de los casos generaban millones de dólares-, sólo con becas de estudio, alojamiento y material académico entre otras remuneraciones. Sin embargo, el Tribunal de California (7/6/2014) decretó una sentencia cuya resolución dejaba la puerta abierta para que desde el año 2016 las universidades, puedan empezar a pagar a sus jugadores de futbol y baloncesto -las dos franquicias con límite de edad-, un máximo de 5.000 dólares por temporada.
} 
efectivo, en este caso, sirve solo para "taponar agujeros". La suma de todos los salarios anuales de los jugadores de cada franquicia, no puede excederse de un cierto límite, ese digamos que es el primer "tope" salarial propiamente dicho. Sin embargo, existen diversos mecanismos conocidos como "excepciones" que permiten superar ese primer tope y se le garantiza a un jugador de determinadas condiciones, cobrar más del mínimo establecido para cada caso. En el supuesto escenario donde los equipos sobrepasen en salarios el "segundo" tope, esto es, no respetar las "excepciones" que las normas de cada competición pactarán, deberán pagar una "multa" a la liga, consistente exactamente en la cantidad que se pasan de ese tope. El modelo americano es un modelo basado en el equilibrio deportivo, el cual intenta igualar la capacidad de gasto de todas las franquicias -sobre todo de aquellas con desigual potencial económico-, fenómeno que viene derivado de la ausencia de mecanismos de reparto de ingresos entre entidades con capacidades muy desiguales para generar recursos, por operar en áreas geográficas y/o mercados dispares, y en donde esta alternativa a la redistribución de recursos se vislumbra en la fijación de unos niveles homogéneos de gasto en salarios medida que contribuye a equilibrar el potencial de los equipos y en consecuencia a ofrecer un producto más atractivo para los consumidores o usuarios de este modelo de negocio. Y la equidad que relatamos es tal en las franquicias americanas, que el monto de dichas multas o sanciones por excederse de esos topes salariales señalados, se reparte entre todos los equipos que no incumplan con dicha norma con idéntica asignación a cada uno de ellos.

En definitiva estamos ante una idea del negocio deportivo autofinanciado, el que impera en el negocio americano, basado en la equidad -equilibrio competitivo- ${ }^{16}$, en el que interesados inversores privados tratarán de obtener el máximo beneficio ofreciendo el mejor espectáculo; mientras que el modelo europeo, lejos de priorizar la rentabilidad económica -pese a que a realizado avances en la gestión profesionalizada de las entidades-, basa su idiosincrasia en el deporte asociativo lúdico entre sus practicantes,

\footnotetext{
16 Entre otros estudios basados en este equilibrio competitivo del modelo americano, destacan; BUTLER, M.R., "Competitive balance in MLB", The American Economist, Vol. 39, No 2, 1995; GUSTAFSON, E., FIZEL, J. y Hadley, L., Baseball Economics, Edit. Praeger, 1997; ForT, R. y QuIRK, J., "Cross subsidization, incentives and outcome in professional team sports leagues", Journal of Economic Literature, $\mathrm{n}^{\circ}$ 33, 1995; SHEEHAN, R.G., Keeping score. The economics of big-time sports, Edit. Diamond Communications, 1996.
} 
en donde se da prioridad a los resultados deportivos por encima de los económicos, y en donde se persigue la profesionalización de los deportistas sin abandonar la preeminencia de lo deportivo sobre lo económico, pero sin estructurarse como una verdadera industria -caso americano-, tendente a la obtención de beneficios.

\section{TENDENCIAS AL MODELO DE NEGOCIO AMERICANO EN EL PANORAMA INTERNACIONAL}

La NBA como modelo de negocio deportivo a seguir fue copiada en mayor o menor medida por muchas ligas del mundo, con mayor o menor grado de éxito ${ }^{17}$. Pero siempre de alguna $\mathrm{u}$ otra manera se vieron forzados a lidiar con las diferentes peculiaridades de cada país, ya sea a nivel legal, económico, o incluso político. Por ejemplo, la PBA filipina, la primer liga profesional de básquet creada fuera de los Estados Unidos (en 1975), es un conjunto de corporaciones que en realidad son secciones locales de compañías internacionales y son las compañías mismas que la publicitan (en su momento la petrolera Shell, Coca-Cola, la Corporación San Miguel, etc.) quienes constituyen los equipos, generan los ingresos propios del equipo, elaboran los contratos y pagan los sueldos, es decir, no son compañías creadas "ad-hoc" para cada equipo. La Lega Italiana di Pallacanestro (llamado comúnmente Lega Basket Serie A) fue, en la temporada 1969-70, la primer liga de Europa en tener sus clubes de primera división agrupados en una asociación de clubes autogestionable, y en el caso italiano con el tiempo devino en sociedad anónima. Si bien su constitución contempla un régimen mixto entre socios que son sociedades y socios que son solo clubes, con el tiempo estos últimos también se constituyeron en sociedades pero por una cuestión más bien de acogerse a las leyes de mercado y competir en igualdad de condiciones. A pesar de que la Lega Italiana es y responde legalmente como una sociedad anónima, ésta, si bien no depende de la Federación italiana, es sometida a muy fuertes regulaciones.

\footnotetext{
${ }^{17}$ No todo el proceso de la implantación del modelo de negocio se ha desarrollado exento de polémica; las decisiones condenatorias de los Tribunales se han sucedido, subrayando unas veces el carácter anticompetitivo del sistema, y otras la naturaleza monopolística de las medidas empresariales unilaterales en este tipo de modelos en lo que respecta a las relaciones contractuales laborales, situando en muchas ocasiones al margen de la legalidad, los acuerdos empresariales que habían regido el deporte profesional durante estos años, así, con el cuestionamiento jurídico de las reglas y normas restrictivas de la NFL, NBA y NHL, llegó la fecha de caducidad de un modelo de mercado de transacción de deportistas regido de manera unilateral ( $C f r$. en este sentido la Sentencia de la Corte Suprema del caso, Robertson $v$. National Basketball Association, 389 F. Supp. 867 (1975)).
} 
Sin embargo el caso estrella a analizar diferente a la NBA es sin lugar a dudas la ACB española. Como la mayoría de las ligas europeas, esta fue creada en base a los equipos que compitieron en la primera división de su Federación el año anterior. El modelo a seguir, si bien fue calcado de la NBA, fue la base que creo el modelo de liga asociación de clubes que después copiaría, entre otras, la Liga Nacional argentina (1985).

En España, la Liga ACB (Liga española de baloncesto), arranco en la temporada 1983-84 como una asociación de clubes - concepto distinto a la franquicia-, no obstante, seria a partir de la temporada 1991-92, con la sanción de la Ley del Deporte española, cuando se produce una revolución en todo sentido. Esta ley obliga a todos los clubes que no tengan superávit en los cinco ejercicios inmediatamente anteriores a convertirse en sociedades anónimas deportivas ${ }^{18}$. El salto cualitativo en la gestión de los clubes españoles se produce cuando estos, a pesar de ese brusco cambio, siguen ejerciendo (e incrementando de manera progresiva) su función social. Es decir, visión 100\% de empresa, sin dejar por ello de ser un club. Por cuanto a la Liga misma, legalmente es una sociedad anónima y sigue el modelo de gestión de la NBA. En el caso puntual de la NBA ninguno de los equipos es un club. Cada equipo se encarga de todos aquellos contratos de difusión y comunicación (radio, televisión, etc.) a nivel local (es decir, municipal -de condado- e incluso estadual), pero la NBA se reserva el derecho de todos los contratos firmados a nivel nacional.

\section{REALIDAD Y FUTURO DE LA FRANQUICIA EN EL FUTBOL.}

Pensamos que la oportunidad del modelo de negocio de la franquicia en este deporte tiene una expansión y un desarrollo igualmente ilimitado. La imagen de marca que en la actualidad tienen los equipos de futbol españoles $-\mathrm{y}$ europeos ${ }^{19}$-, es tal, que se

\footnotetext{
${ }^{18}$ El régimen jurídico específico de las Sociedades Anónimas Deportivas (SAD) se encuentra en los artículos 19 a 29 de la Ley 10/1990, de 5 de octubre, del Deporte, y en el Real Decreto 1251/1999, de 16 de julio, sobre sociedades anónimas deportivas, modificado por el Real Decreto 1412/2001, de 14 de diciembre.

${ }^{19}$ Vid. Terol Gómez, R., Las Ligas ... op. cit., pág.54.
} 
hace imprescindible el desarrollo de nuevas formulas jurídicas en los países donde no se practique tanto este deporte, o incluso, donde este deporte sea ignorado como competición deportiva, al existir en el mismo otro tipo de actividad que prime por encima del futbol, y en este sentido el modelo de negocio de la franquicia, a la postre, se podría convertir en acicate para que potenciales emprendedores decidan implantarlo y a su vez, atraigan a accionistas o propietarios para que desarrollen y expandan su marca y su modelo de negocio desarrollado.

Estableciendo una comparativa con la Mayor League Soccer (MLS), de EE.UU, nos damos cuenta que el futbol europeo está casi destinado al fracaso si no se modifica la dedicación y profesionalidad de los responsables. Las competiciones europeas llevan unas políticas financieras auténticamente ruinosas (solo en la Liga española en la temporada pasada 2013 la deuda fue de 2.000 millones de euros, en la actualidad). ${ }^{20}$ Inexcusablemente todo esto cambiará no ya solo el día que Europa le eche el freno al fútbol español, sino en el mismo momento en el que estas propias sociedades anónimas deportivas dejen de pensar exclusivamente en los éxitos deportivos y se dediquen a mirar un poco el apartado de los beneficios. Es alarmante que se permitan fichajes estratosféricos en los que "supuestamente" se desembolsan cantidades ingentes de dinero (así no es de extrañar que en los últimos años hayan aterrizado en nuestro fútbol

\footnotetext{
${ }^{20}$ A Junio de 2014 la deuda de la Primera División Española es de 3.15 billones de euros, y la del futbol profesional en total más de 5 billones de euros. De estos cerca de 2 billones son debidos al Estado entre Impuestos y Seguridad social, y el resto fundamentalmente a los Entidades Bancarias. (Los datos utilizados se han obtenido a partir de http://empolicalcio.forumfree.it/?t=46542599\&st=45 y la última Global Sports Salaries Survey disponible en www.sportintellingence.comhttp://www.csd.gob.es/csd/estaticos/notici...rio-csd-lfp.pdf)). Pero igualmente los precedentes deficitarios para las Administraciones Públicas en Europa has sido múltiples, recordamos a modo de ejemplo el apoyo financiero de las Administraciones a los Clubes y Sociedades Anónimas Deportivas que se ha producido de diversas maneras a lo largo de los años, desde la participación en el accionariado de las sociedades anónimas (como fueron los casos del Racing de Santander, Jaén, entre otros equipos), hasta el otorgamiento de avales públicos para garantizar las operaciones de crédito o préstamo destinadas a pagar las deudas contraídas (caso del aval del Gobierno Foral de Navarra en 2003, Ley Foral 1/2003, de 14 de febrero, a favor del Club Deportivo Osasuna, por cuantía de 18 millones de euros), alquileres de instalaciones deportivas públicas a precio simbólico [Alavés, Celta, Deportivo, Villarreal o Mallorca no abonaban un solo euro a las arcas públicas por la cesión de sus campos de fútbol, y otros como Rayo Vallecano o Málaga pagaban cantidades simbólicas). Respecto a Europa por ejemplo recordamos igualmente entre los muchos acontecimientos no deseables en este ámbito, el caso italiano, cuyo Parlamento, mediante la Ley 27 de 21 de febrero de 2003 (Ley conocida como Salva Calcio), aprobó una serie de medidas fiscales y contables para clubes profesionales investigadas por la UE, por posible infracción del artículo 87 del Tratado, sobre ayudas de Estado., que llevaron al Estado aportar cantidades millonarias para la salvaguarda de la competición.
} 
los jeques provenientes de los países más ricos del planeta) ${ }^{21}$. Sin embargo la MSL, la Liga americana basada en un modelo de negocio franquiciado, un negocio con sus defectos y fortalezas pero que lleva una estricta política financiera y, debida al límite salarial establecido, sus franquicias sólo pueden pagar elevadísimas cantidades de dinero a un número determinado de jugadores: concretamente, sólo 3 por equipo pueden pasar de los cerca de $\mathbf{4 0 0 . 0 0 0}$ dólares anuales. Y como toda cifra que supere ese límite debe ser abonada íntegramente por la franquicia (el resto de sueldos están garantizados por la Liga y se pagan de un fondo común), y no todas tienen la misma capacidad para generar ingresos, la mayoría de franquicias prefieren mantenerse en un discreto segundo plano en materia salarial.

En la actualidad el paso más relevante que dio el futbol español para afianzarse en el mercado norteamericano, aparte de las giras internacionales, fue el intento del FC Barcelona de convertirse en propietario de una franquicia de la Major League Soccer $(\mathrm{MLS})^{22}$. En mayo de 2008, el FC Barcelona firmó un contrato de partnership (contrato

${ }^{21}$ Vid. RUBIO SÁNCHEZ, F., El contrato de trabajo de los deportistas profesionales, Dykinson, 2002.

22 La MLS otro modelo de negocio similar basado en el nuevo modelo de franquicias, sigue creciendo en equipos, calidad, estrellas, y por supuesto, en jugadores "franquicia", esto son jugadores que según el Convenio Colectivo permiten que cada franquicia -Club-, designe alguno de ellos para cada temporada, y han de estar sujetos a un régimen de agente libre restringido en los términos que a continuación se señalan; si en la temporada en que finaliza su contrato mantiene la consideración de jugador franquicia, el Club podrá obtener una compensación consistente en dos primeras rondas siempre que le ofrezca renovar proponiéndole al menos un salario equivalente al de la media de los cinco jugadores de su mismo puesto mejor pagados durante la temporada que finaliza; y si tal oferta no se produce, el jugador adquiere la condición de agente libre sin ningún tipo de restricción, disponiendo de absoluta libertad para negociar con otra franquicia. En la actualidad (temporada 2014/15) la Liga profesional de futbol estadounidense cuenta con una cifra record de 25 jugadores franquicia, cada vez se va adaptando el modelo de negocio permitiendo la contratación de jugadores más jóvenes e implementando nuevas reglas para facilitar la contratación de jugadores con más potencial de futuro. Así tras la actual reforma de la normativa actual para la presente temporada se contempla la contratación de jugadores internacionales Sub'20, al igual que establece el sueldo máximo que se puede pagar a un jugador - no franquicia-, que asciende a 387.500 dólares, es decir por mucho que se esté pagando a un jugador franquicia -para los cuales no existe tope salarial excepcionalmente-, para el presupuesto del tope salarial del club sólo contará ese jugador, esto es, solo computará por valor de 387.500 dólares. La Ley de Jugador Franquicia (conocida como la Ley Beckham ya que fue aprobada por la MSL para que el jugador inglés se pudiera incorporar al L.A Galaxy en 2007), se estableció sin romper con los pilares del modelo de negocio basado en el tope salarial -Beckham, fue el Jugador Franquicia de los Galaxy con un sueldo de cerca de 8 millones de dólares anuales-, ya que su salario solo computaba en el presupuesto del Galaxy para la temporada como 350,000 dólares (que era el tope salarial fijado en 2007). En La norma vigente del Jugador Franquicia les permite a cada una de las franquicias contratar hasta tres jugadores con salarios que superan el tope salarial del club y cuyo costo adicional será responsabilidad financiera del club - de la franquicia que lo contrató-, y no de la Liga que asumirá el de los demás, y el mismo pero sólo por valor del tope. Los cupos para los Jugadores Franquicia 
de asociación), para trabajar de manera estratégica con la MLS, según el cual el club catalán disputaría en EEUU seis partidos entre 2009 y 2013, tendría presencia entre los patrocinadores de la liga, podría firmar acuerdos comerciales bilaterales con empresas americanas y podría optar a una franquicia a partir de $2010^{23}$. El 15 de octubre de 2008, el FC Barcelona y un grupo inversor liderado por el boliviano Marcelo Claure presentaron una oferta para convertirse en nuevos propietarios de una de las nuevas franquicias, localizada en Miami. No obstante, el crac económico americano del 2009 y la inestabilidad generada hicieron que el 3 de marzo de 2009 la MLS, el FC Barcelona y Marcelo Claure acordasen "desestimar la candidatura de Miami como nueva franquicia en procesos de expansión de la MLS". ${ }^{24}$

Aunque la franquicia del FC Barcelona en Miami fue el proyecto más avanzado hasta el momento para que un club español entrase a competir en el sistema de competiciones cerrado americano, en 2006 el Real Madrid firmó un precontrato con la NBA para adquirir una de las cuatro franquicias europeas que la liga americana planifica en el viejo continente ${ }^{25}$ y cuatro años atrás, el FC Barcelona fue el primer club

\footnotetext{
pueden ser usados para adquirir jugadores nuevos en la MLS o retener jugadores actuales en la MLS, pero todo -y he aquí el funcionamiento del modelo de negocio deportivo americano-, sujeto a la aprobación de la Liga no de la propia franquiciada. Curioso es también dentro de este sistema y en su regulación, los denominados jugadores "lotería", jugadores de estimada valía asignados a clubes de la MLS siguiendo el proceso de lotería, pero con la peculiaridad que al equipo que se le asigna un jugador por medio de la lotería en cualquier temporada no le será asignado otro jugador por medio de este mecanismo esa misma temporada a menos que o hasta que todos los clubes hayan recibido un jugador por lotería o que algún club haya decidido no participar en una lotería -como vemos, impera en todo momento el principio de equidad-. Evidentemente este tipo de jugadores franquicia que podrán utilizar cada uno de los clubs pertenecientes a la competición, no garantiza el campeonato, pero cumplen con el binomio y máxima del modelo de negocio americano, espectáculo-rentabilidad, ya que los beneficiarios -consumidores y usuarios-, compraran sus entradas y llenarán sus estadios, provocando el éxito de la competición, de las franquicias, de la Liga y del consumidor final.
}

Cfr. FC. BARCELONA, "Asunto: El Barça desestima la candidatura de la MLS a Miami”, nota informativa, 3-3-2006.

${ }^{23}$ Cfr. GINESTA PORTET, X., "Football and the Global Entertainment Business. Clubs as Entertainment Multinational Corporations", Communication \& Society 24 (1), 2011, págs.141-166.

${ }^{24}$ Cfr. LEE, F., "Spectacle and Fandom: Media Discourse in Two Soccer Events in Hong Kong", Sociology of Sport Journal, n 22, 2005, págs.194-213.

${ }^{25}$ Cfr. ZÁRATE, Óscar, “Motín contra Calderón”, Mundo Deportivo, 12-12-2006, pág. 17. 
de fútbol vinculado con la National Football League (NFL) americana, ya que absorbió como sección del club a los Barcelona Dragons ${ }^{26}$.

\section{VII.-EL VALOR CONTINGENTE DE LA FRANQUICIA: LIGA ACUAL DE FUTBOL INDIA.}

En la actualidad, esta actitud empresarial o iniciativa de negocio abanderada por el concepto de franquicia, es la que acaba de implantarse en la India. Efectivamente en el presente 2014, ha surgido la Indian Super League, (liga india de futbol), liga de nueva creación que ofrece un modelo de negocio cuyo principal revulsivo para los nuevos inversores, son los importantes incentivos fiscales que proponen. Esta, pretende imponer en el mercado nuevas fórmulas jurídicas creadas para atraer a los equipos de las ligas europeas y que se conviertan en accionistas de las propias franquicias futbolísticas en este país. Uno de los equipos españoles que primero ha decidido vincularse a este modelo de negocio ha sido el Atlético de Madrid, que tras un periodo de negociación contractual con la propia Liga India, y fruto de esas negociaciones, llega a poseer en la actualidad, el 25\% del Atlético de Kolkata (franquicia India), con el que, entre otras muchas cosas como el staff corporativo, organigrama, jugadores, etc., comparte colores -equipación-, por tanto, se ha convertido en su franquicia en esta competición india.

Este negocio del mercado indio, sus pretensiones y los proyectos que ambicionan, pretenden que dicho país tenga una liga de futbol a la altura de las mejores competiciones europeas -y por ende, del mundo-. El desarrollo del modelo o sistema comercial que han puesto en vigor y en la actualidad están desarrollando (en concreto el 15 de septiembre de 2014, se inició la Liga de futbol India), empieza por intentar implicar a los equipos europeos para que participen como accionistas en sus equipos, y para ello presentaron una serie de formulas legales que permitían asociaciones y colaboraciones entre los equipos europeos y la propia Liga que debían fructificar y materializarse para que finalmente dichos acuerdos dieran lugar a transformaciones en acciones de los equipo de la Liga india (Indian Super League) ${ }^{27}$.

\footnotetext{
${ }^{26}$ Cfr. Mundo Deportivo, "El Barça entra por la puerta grande en EEUU”, 1-2-2002, pág. 16.

27 Esta liga esta promovida por Reliance, la mayor empresa privada del país, y por una filial de $21 \mathrm{st}$ Century Fox. En la que cada equipo cuenta con el apoyo económico de una compañía y de un actor de Bollywood o deportista famoso. Los equipos indios buscan el apoyo técnico de clubes europeos para poner en marcha la liga profesional y los salarios tributan a un tipo lineal del $20 \%$ en el IRPF, beneficiando a los contratos estrella de los jugadores.
} 
Las formas jurídicas de colaboración o asociación propuestas fueron varias, así por ejemplo, la Florentina (afamado club italiano de futbol), acaba de vincularse con el equipo Indio, el "Pune", mediante un contrato donde la parte contratada -club italiano-, prestara servicios de asesoramiento gratis durante el primer año, y si ambas partes lo consistiesen y el negocio fructificase, se fijaría un canon -precio-, por ese trabajo y en el segundo ejercicio se convertiría en accionista de la franquicia del club indio.

Esta fórmula es absolutamente novedosa desde el punto de vista legal deportivo, pues lo habitual es que un equipo se convierta en accionista de otro o incluso que cobre directamente una remuneración a cambio de prestar servicios de asesoramiento ${ }^{28}$. Este tipo de acuerdos tienen más semejanzas con el sistema de franquicia americano que los que se realizan en Europa. ${ }^{29}$

El principal atractivo de entrar en estas franquicias radica, por un lado, en su alto potencial de público, lo que puede servir para atraer patrocinadores que quieran abrirse camino en este mercado, mientras que por otro, los precios son atractivos ya que se trata de una liga que al estar en su fase inicial y ser novedosa y pionera en su sector, todavía esta a precios asequibles y por tanto, al alcance de todos los clubes europeos. Si se consolida -como ocurrió con la Liga de Cricket-, y tiene la recepción prevista, el ser dueño de una franquicia en India puede convertirse en un negocio muy rentable. ${ }^{30}$

\footnotetext{
${ }^{28}$ En la actualidad se acaba de presentar este modelo de negocio en la ciudad de Nueva York con la incorporación de una nueva franquicia futbolística, fruto del acuerdo de colaboración realizado por el Manchester United -equipo inglés-, donde ha surgido la nueva franquicia "New York City Football Club", que es el nuevo equipo de fútbol profesional de la liga de futbol de Estados Unidos, de la ciudad de Nueva York. Fue fundado en 2013 por el Manchester City y los New York Yankees, quienes conjuntamente pagaron 100 millones de dólares para incorporarse como nueva franquicia en la Major League Soccer de los Estados Unidos a partir del año 2015. Y donde uno de nuestros jugadores más emblemáticos de la Selección española va a jugar la presente temporada en dicha franquicia, David Villa.
}

${ }^{29}$ La competición, como señalamos pionera dentro del modelo de negocio futbolístico, se ha iniciado en el mes de septiembre de 2014, finalizando en el mes de diciembre del presente año, ya que en la actualidad su modelo de negocio deportivo esta formado únicamente por ocho equipos, pero con un potencial muy relevante, ya que el mercado indio tiene un atractivo espectacular y un valor absolutamente al alza, pues su mercado cuanta con cerca de 1.200 millones de habitantes. Creemos rotundamente que esta iniciativa podrá ser acicate para que dicho negocio se extienda por otros lugares y continentes del mundo.

\footnotetext{
${ }^{30}$ Aún no existen cálculos oficiales, pues se esta desarrollando la Liga en el presente año 2014, pero ya se habla de cifras multimillonarias, y sobre todo teniendo en cuenta que la FIFA igualmente invertirá en los futuros estadios que se construirán en India, ya que será el país anfitrión del Mundial Sub-17 que se celebrará en 2017.
} 
Independientemente del atractivo de este mercado, observamos las peculiaridades legales de estos acuerdos para desembarcar en la Indian Super League. Aunque el país cuenta con muchos incentivos de tipo fiscal, los equipos deberán de estudiar -y en ello están-, en la mejor manera de articular el acuerdo contractual, por ejemplo a la hora de tributar eligiendo entre pagar el 12\% de IVA o el 20\% de IRPF, tipo único que se aplica en estos $\operatorname{casos}^{31}$. A pesar de estas facilidades, también existen barreras, como las restricciones en las cantidades de dinero que se pueden enviar al país, por lo que un accionista tiene que valorar cuáles son las mejores alternativas para fichar a un jugador estrella.

Por ahora, las alternativas contractuales que se articulan para participar en la competición india, se desarrollan de tres formas distintas:

1.- La primera, y elegida recientemente por el Atlético de Madrid, es consentir un contrato que le vincule al proyecto como accionista.

2.- El segundo acuerdo o alternativa para formar parte de la competición, lo emplea la Florentina, que en este caso ofrece apoyo técnico gratis y asume el coste como si de una campaña de marketing se tratara.

3.- Y por último, existe un modelo híbrido, en el que además de soporte técnico, se puede incluso ceder jugadores o al entrenador y, en el futuro, contabilizar esa contribución y canjearla por acciones.

El modelo de negocio impulsado por India, a pesar de que por el momento es poco habitual que un equipo extranjero invierta en otro club y, mucho menos, que se haga fuerte en otras ligas a través del modelo de negocio de franquicia, pensamos que podrá suponer un cambio de tendencia en el mundo del futbol, y podrá ser un revulsivo para intentar acometer por medio del deporte, nuevos yacimientos de empleo, nuevas

${ }^{31}$ Cfr. SAIZ, S., "India tiende un puente legal a los clubes españoles de fútbol”, Diario Expansión, 18 de agosto 2014, Madrid. 


\section{Doctrina y Jurisprudencia}

iniciativas empresariales y a su vez satisfacer nuevas necesidades de entretenimiento y acceso a actividades deportivas, y por ende, saludables para consumidores y usuarios.

\section{REFLEXIÓN FINAL}

A modo de conclusión, debemos entender que parte del concepto fundamental de global es, en términos simples, la desaparición de cualquier elemento que dificulte la interacción. Las empresas buscan comercializar globalmente o en lenguaje menos complicado, a escala internacional o mundial. La globalidad en este sentido, nos hace recordar a los mercaderes europeos buscando, en glamurosas aventuras, llevar exóticos productos de un lugar a otro para obtener más y mejores ganancias. Vemos que las fusiones, adquisiciones o alianzas estratégicas pretenden explotar mejorar los mercados, y en este contexto, las franquicias están creciendo en todos los sectores empresariales y en todas las naciones libres del mundo, son métodos y modelos de negocio cada vez más populares de distribución internacional. La globalización ya no es -si es que alguna vez lo fue-, una simple percepción de las cosas, es una realidad, es un proceso que algunos rechazan y otros aceptan, $\mathrm{y}$, muchos abrazan, pero que se ha convertido en el nuevo entorno de los negocios, significa que un producto, servicio o concepto creado en casi cualquier país, puede ser consultado o implantado en otro. La globalización genera un nivel de competencia en el mercado que la mayoría de los empresarios pequeños y medianos difícilmente podrán soportar por sí solos. La franquicia ha llevado no solamente modelos de negocio a través de sus productos, servicios y conceptos comerciales a prácticamente todo el mundo, sino que ha difundido culturas y estados de vida que, tarde o temprano, terminan por ser aceptadas y adoptadas por otros mercados como propios.

En efecto, ni el sometimiento de las entidades participantes en competiciones calificadas como profesionales, fútbol $1^{\mathrm{a}}$ y $2^{\mathrm{a}}$ división y baloncesto $\mathrm{ACB}$, a un régimen de responsabilidad mercantil a través de la particular figura de las Sociedad Anónima Deportiva (SAD), ni la progresiva profesionalización en la gestión del deporte profesional, ni el ingenio y el esfuerzo dedicados a la búsqueda de nuevas fuentes de financiación han paliado los problemas de origen estructural que padece el modelo de 
deporte profesional en el Estado Español y en el conjunto de Europa ${ }^{32}$. En el eje de la crisis del modelo europeo, se sitúa igualmente el diseño disfuncional de las relaciones laborales deportivas y del mercado de trabajo. El factor humano, los deportistas, son el elemento decisivo para lograr un producto deportivo atractivo, esto es, equilibrado, y por tanto exitoso. En efecto, una asignación homogénea de los talentos deportivos, como condición de un producto con incertidumbre sobre el resultado, es fundamental para la actividad empresarial deportiva si se pretende que sea próspera.

Por lo tanto creemos que la organización de las competiciones requiere de reglas tendentes a promover la constitución de plantillas deportivas igualadas, o lo que es lo mismo, se precisa la intervención conjunta sobre la asignación de recursos humanos entre las entidades deportivas, más aún cuando el potencial económico de estas, y en consecuencia su capacidad de atraer y contratar a los mejores deportistas, es dispar. Y en este sentido, el sistema de franquicia no tiene otro camino más que el éxito, no son la panacea a los problemas económicos de las personas ni de los clubs ni de los equipos deportivos, ni mucho menos son la única forma de desarrollarse en el mundo económicamente cambiante al mundo en que vivimos, pero sí, hoy por hoy, son una de las mejores opciones para hacer negocios como hemos observado, incluso en el terreno deportivo. Los mercados nacionales y extranjeros están sedientos de negocios innovadores, globalizados, y las franquicias, pese a las fuertes crisis, siguen y seguirán adelante, generando beneficios, fomentando la investigación y el desarrollo, fomentando la competencia y reducción de precios, colaborando en la creación del tejido empresarial, fomentando empleo, haciendo crecer a la economía, y sobre todo, contribuyendo con el desarrollo de las comunidades, y de las naciones enteras, y a la generación de beneficios para los consumidores y usuarios finales, beneficiarios de dichos modelos de negocio. Las medidas a adoptar deberán tener un alcance general, comprensivo al menos de las Ligas europeas de primer orden, ya que en un mercado de trabajo deportivo cada vez más globalizado, carece de sentido confinar las soluciones a compartimentos estancos. Y aquí, el papel promocional de la UE adquiere una importante dimensión, a la hora de impulsar y consolidar a los agentes sociales europeos del sector deportivo, acelerando el

\footnotetext{
${ }^{32}$ Cfr. IRURZUN UGALDE, K., "La negociación colectiva en el deporte profesional”, Universidad del País Vasco, Leioa, 2004.
} 
actual proceso de Diálogo Social abierto entre los distintos operadores económicos y deportivos. 